\title{
EL LIDERAZGO EN LA GESTIÓN DE LA CALIDAD DE LA EDUCACIÓN A DISTANCIA COMO INNOVACIÓN
}

\author{
(LEADERSHIP IN THE MANAGEMENT OF QUALITY OF DISTANCE EDUCATION AS AN \\ INNOVATION)
}

J osé Silvio

Nova Southeastern University (EE.UU.)

\section{RESUMEN}

El objetivo principal de este trabajo es contribuir a un mayor y mejor conocimiento sobre el ejercicio de un liderazgo efectivo con miras a lograr una mejor gestión de la calidad de la educación a distancia y de su difusión en la sociedad como innovación. En la primera parte, se definen algunos conceptos básicos y relevantes para el logro del objetivo enunciado. En la segunda parte, se discute cómo se relacionan esos conceptos entre si y se integran en un marco de referencia para el ejercicio de un liderazgo efectivo para la gestión de la calidad en la educación a distancia como innovación.

\begin{abstract}
The main objective of this paper is to contribute to the achievement of a better and increased knowledge on the exercise of leadership, with the aim of improving the management of the quality of distance education, and its diffusion in society as an innovation. In the first part, some basic and relevant concepts to achieve the above mentioned objective are defined. In the second part, we discuss how those concepts relate between them and integrate in a framework to promote the development of an effective leadership in managing the quality of distance education as an innovation.
\end{abstract}

\section{INTRODUCCIÓN}

El sistema educativo en los países comúnmente llamados en vías de desarrollo, y en especial el destinado a la educación superior, pasó mucho tiempo en un ambiente relativamente plácido, en el cual nadie se interrogaba acerca de su calidad. Se daba por sentado que en ellos se impartía una educación de alta calidad. Nadie cuestionaba la calidad de esas instituciones y al referirnos a esas universidades, la palabra calidad era casi inexistente en el lenguaje académico. Nadie pensaba que era necesario crear sistemas para evaluar la calidad y acreditar programas y organi- 
zaciones educativas. Pero, con el tiempo, han ocurrido varios hechos que han perturbado esa tranquilidad y placidez. Uno de ellos fue el proceso de masificación de la educación superior, que obligó a las universidades a abrir sus puertas a una mayor y más variada población estudiantil.

Pero, más adelante ocurrió otro hecho significativo: la aparición de la educación a distancia. Al principio, la educación a distancia surgió como una solución remedial a dos problemas. En primer lugar, proveer educación para contribuir a canalizar la demanda educativa masificada y atenuar la presión de la masificación sobre las universidades presenciales. En segundo lugar, ofrecer oportunidades educativas a la población incorporada al sistema laboral, la cual por diversas razones no pudo aprovechar esas oportunidades en universidades presenciales. Sin embargo, la aparición de la educación a distancia por si sola no tuvo una influencia determinante sobre la calidad. La educación a distancia era al principio portadora no sólo de una esperanza para muchos, sino de un nuevo paradigma de enseñanza y aprendizaje, basado en la libertad, la facilitación y el constructivismo del aprendizaje, en oposición al directivismo. Sin embargo, la dependencia de la educación a distancia de los medios tradicionales de comunicación la fue progresivamente convirtiendo en una educación más, considerada por muchos de segunda. Esto mantuvo la preocupación por la calidad en un estado todavía latente. Lo que realmente está revolucionando la educación distancia es la aparición y el desarrollo de la informática y la telemática y sus aplicaciones a la educación. Ahora, ya es posible hacer realidad el ansiado paradigma de educación libertaria, facilitadora y constructivista del aprendizaje, el aprender de diversas maneras, en cualquier lugar y en cualquier momento, rompiendo las barreras de espacio y tiempo, que antes restringían a los actores implicados en el proceso educativo.

Esta nueva edición de la educación a distancia, acompañada ahora de la virtualidad y las poderosas tecnologías digitales de información y comunicación, amplía y profundizan las oportunidades para muchos, pero también son amenazas para otros. Ahora la gente empieza a preocuparse por una nueva modalidad educativa, que puede crecer y diversificarse sin ningún control de calidad. Pero esta preocupación también se ha extendido a la educación presencial llamada tradicional y por extensión ha llegado a la educación a distancia. A ello se agregan otros factores, a saber: la creciente presión por parte de las autoridades gubernamentales, de exigir un mayor control sobre los recursos asignados a la educación superior y el requerimiento de rendición de cuentas a la universidades financiadas con fondos públicos de los gobiernos centrales nacionales. A esto se agrega una proliferación de nuevos proveedores de educación superior, tanto del mundo académico como no académico tales como, empresas, organizaciones no-gubernamentales y otras, que utilizan la educación virtual y a distancia como un instrumento para ofrecer servicios educativos no-tradicionales a una nueva población más numerosa y diversa que necesita aprender de otra forma, más flexible. La educación como tal está descubriendo la calidad, lo que la industria ha descubierto hace mucho tiempo y muy lentamente se está despertando y movilizando para hacerla suya. Todos estos hechos convergen para crear una masa crítica de factores y un ambiente apropiado para evaluar la calidad tanto de la educación presencial como de la educación vir- 
tual y a distancia, acreditar programas e instituciones oferentes de ambas modalidades educativas y mantener la calidad de esa educación. Aquí intentamos responder a esta última necesidad, la de mantener la calidad. Para mantener es necesario gestionar adecuadamente todos los elementos que contribuyen a producir calidad en la educación a distancia, sobre todo tratándose de una innovación como la educación virtual y a distancia. Toda gestión necesita líderes que la conduzcan y especialmente cuando el objeto de la gestión es una innovación la cual hay que difundir y mantener en la sociedad a un nivel elevado de calidad para evitar su deterioro.

\section{ALGUNOS CONCEPTOS BÁSICOS}

\section{Educación virtual y a distancia}

La virtualización es un proceso y resultado al mismo tiempo del tratamiento y de la comunicación mediante computadora de datos, informaciones y conocimientos. Más específicamente, consiste en representar electrónicamente y en forma numérica digital (mediante unos y ceros) objetos y procesos que encontramos en el mundo real. En el contexto de la educación, la virtualización puede comprender la representación de procesos y objetos asociados a actividades de enseñanza y aprendizaje, investigación y gestión, así como objetos cuya manipulación permite al usuario, realizar diversas operaciones a través de INTERNET (Silvio, 2000).

Pero, la precisión de los conceptos se advierte mejor en el contexto de la tridimensionalidad en la cual se mueve la educación actual, en el cual se relacionan el espacio, el tiempo y la virtualidad. El espacio y el tiempo son las dimensiones generales en las cuales transcurren los procesos de enseñanza y aprendizaje. Para nuestros propósitos, los actores que intervienen en dichos procesos pueden estar en el mismo espacio (o lugar) en espacios diferentes, pueden interactuar y comunicarse al mismo tiempo (de manera sincrónica o en tiempo real) o en tiempos diferentes (comunicación asincrónica o diferida) Con respecto a la virtualidad, que es una característica producto del proceso de virtualización, los actores pueden relacionarse en un ambiente de alta virtualidad o virtualización o de baja virtualidad. El aprendizaje se realiza en un ambiente de alta virtualidad cuando la mayor parte de las actividades, se efectúan a través de computadoras interactuando con un CDROM o a través de INTERNET o de una INTRANET de una organización. La baja o ninguna virtualidad se manifiestan cuando el aprendizaje se realiza de la manera presencial tradicional que conocemos. Pero, si vamos más allá, las combinaciones de los valores de estas tres variables, permiten definir diversas situaciones de enseñanza y aprendizaje en las cuales se manifiestan el aprendizaje virtual y no-virtual y el presencial y a distancia, como se puede ilustrar en la Figura 1.

Cada modalidad de comunicación educativa resultante de cada combinación de espacio, tiempo y virtualidad presenta una característica distintiva particular. No voy a describir cada una de las combinaciones posibles sino las más significativas, para lo referente a la articulación entre el aprendizaje virtual y no-virtual. En primer lugar, encontramos la educación no-virtual presencial, en la cual todos los 
actores están presentes al mismo tiempo en el mismo lugar y caracteriza lo que conocemos como educación tradicional, en la cual no hay ninguna virtualidad. Segundo, la educación no-virtual a distancia, que ejemplifica la educación a distancia tradicional, en la cual educador y aprendiz se hallan en espacios y tiempos diferentes y utilizan soportes muy variados de información no-digitales y no-numéricos ni computarizados y heterogéneos, en un ambiente con ninguna virtualidad, ni ningún recurso electrónico de información. En tercer lugar, la educación virtual presencial, que consiste en actos educativos realizados mediante computadora, pero todos los actores se encuentran en el mismo lugar y al mismo tiempo, como en un laboratorio de computación, por ejemplo, donde todos los actores están relacionados mediante una red local. En este caso, hay una virtualidad elevada al igual que una alta presencialidad y una posibilidad de relación cara a cara entre los actores. Por último, la educación virtual a distancia, en la cual los actores interactúan a través de representaciones numéricas digitales de los elementos del proceso de enseñanza y aprendizaje, en espacios y tiempos distintos. Esta es la modalidad educativa de comunicación asincrónica más reciente y del mayor grado de virtualidad, que se asocia generalmente con el e-learning. Estos cuatro casos ilustran tipos alrededor de los cuales se tejen diversas variantes específicas. Las combinaciones particulares que adopten estas variables están estrechamente relacionadas con los objetivos, contenidos, las metodologías, los recursos y otros elementos de los procesos de enseñanza y aprendizaje.

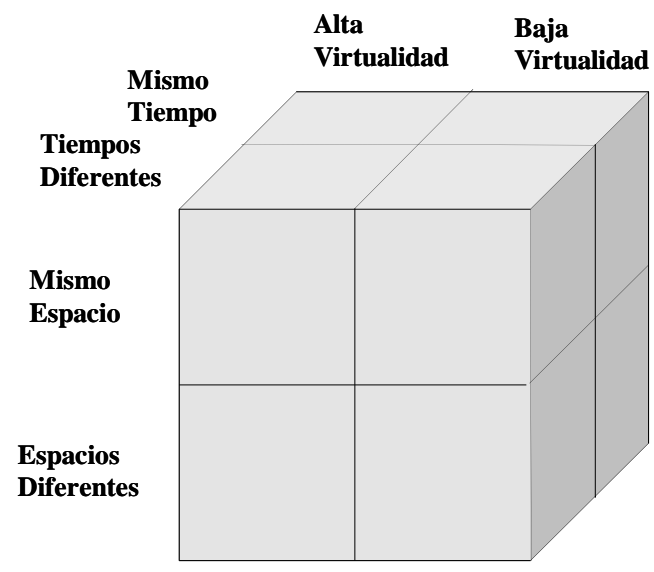

Figura 1. Espacio, tiempo y virtualidad en el aprendizaje

En un contexto menos factual y más normativo, hay diversas maneras de concebir lo que deben ser las relaciones entre la educación virtual y no virtual, por una parte, y presencial y a distancia, por la otra. Esas maneras de concebir conducen a diversos enfoques, que pueden influenciar las estrategias de enseñanza y aprendizaje que se utilicen. Hay quienes piensan que el aprendizaje virtual ha venido a sus- 
tituir al aprendizaje presencial y no-virtual. Se trata entonces de decidir si se adopta uno u otro, pero no articular uno con otro. Luego, encontramos quienes consideran que el aprendizaje virtual debe ser una duplicación del no-virtual. Sobre la base de esta concepción, la educación virtual debe reproducir su homóloga en el mundo real y crear programas educativos en el ciberespacio similares a los presenciales y no-virtuales. Aquí como en el caso de la substitución tampoco hay integración o articulación. Entre estos dos extremos encontramos concepciones y actitudes intermedias, que favorecen la articulación. Entre ellos se encuentran quienes conciben el aprendizaje virtual como un instrumento auxiliar y complementario del presencial y no-virtual. Aquí hay al menos una forma rudimentaria de articulación, pero el aprendizaje presencial y no-virtual sigue siendo predominante y el factor conductor del proceso. Por último, una fórmula más conciliadora, según la cual el aprendizaje virtual debe articularse con el no-virtual, no de una manera fija e inmutable, sino flexible y variable según las necesidades y situaciones (Levy, 1997). La articulación supone también que todas las modalidades educativas se encuentran en igualdad de condiciones, ninguna predomina sobre la otra. Este último enfoque es el que sostengo y el que sirve de base al contenido de este trabajo. El ejercicio combinatorio mediante el cual hemos relacionado el espacio, el tiempo y la virtualidad, está inspirado en la idea y el deseo de articular en vez de sustituir, duplicar o instrumentalizar y de incluir y valorizar, en lugar de excluir y desvalorizar posibilidades, tecnologías, medios tecnológicos y recursos de enseñanza y aprendizaje. La articulación es la única forma posible de aprovechar lo viejo dentro de lo nuevo y resolver los problemas creados por la nueva solución, evitando caer en extremismos y crear una forma armónica de aprendizaje basado en la articulación e integración sinérgica de distintas modalidades educativas. La situación deseable para profesores y estudiantes es poder enseñar y aprender en ambientes de alta virtualidad, espacios y tiempos similares o diferentes, es decir, ambientes donde predomina la articulación de diversas modalidades educativas y se enseña y aprende de manera flexible. La tarea de un administrador y un directivo consiste en realizar una gestión adecuada de esa educación articulada. Los conceptos de virtualización y articulación y las prácticas que de su aplicación a la realidad se derivan, son clave para toda persona que aspire realizar una gestión adecuada de la calidad de la educación a distancia, pues es el ambiente típico en el cual se va a desenvolver. La articulación educativa es un factor importante de calidad de la educación virtual y a distancia.

\section{Calidad}

La calidad se refiere a la conformación de las características de un objeto, material o inmaterial con una norma, criterio o patrón. El término objeto se utiliza aquí de manera muy amplia e incluye los objetos materiales propiamente dichos, los servicios, las ideas, la información y toda producción humana cuya calidad pueda ser susceptible de evaluación. La educación a distancia sería un objeto inmaterial o intangible, cuyo producto son conocimientos adquiridos o aprendidos por personas en ambientes formales o informales y con diverso grado de estructuración. La edu- 
cación puede tener algunos componentes materiales y tangibles, como, por ejemplo, libros y materiales educativos en soportes audiovisuales, locales, edificaciones y laboratorios donde se realiza la enseñanza y el aprendizaje. Pero al evaluar estos materiales pedagógicos, edificaciones y laboratorios lo que nos interesa es su valor para transmitir conocimientos y facilitar su adquisición por parte de seres humanos. De acuerdo a lo anterior y en función de las características del objeto a ser evaluado, hay una evaluación directa y una evaluación indirecta de la calidad. La evaluación directa consiste en evaluar el producto último de un sistema. Por ejemplo, al evaluar un sistema educativo, la medida última de su calidad es el aprendizaje logrado por quienes utilizan sus recursos. Es decir, si los conocimientos adquiridos por los aprendices se conforman con los estándares de calidad establecidos como ideales en nuestro modelo de calidad, se considerará que han recibido una educación de alta calidad. En síntesis, la evaluación directa contempla obtener la información sobre la calidad del sistema educativo, a partir de los portadores directos de los resultados del sistema o los usuarios finales de los servicios del sistema. La evaluación indirecta consiste en evaluar los elementos que el sistema utiliza para funcionar y producir y facilitar el aprendizaje. A partir de esta evaluación y según sus resultados se realizan inferencias sobre la posible calidad de la educación que puede ofrecer. Por ejemplo, si una Universidad posee profesores altamente cualificados, buenas bibliotecas, buenos laboratorios y servicios estudiantiles, podemos concluir que esta Universidad es de alta calidad a partir de los valores de sus elementos estructurales y funcionales y su capacidad para producir un aprendizaje de alta calidad. Esa evaluación es indirecta porque es una inferencia de lo que el sistema podría producir utilizando sus recursos y poniéndolos a disposición de sus usuarios directos, los estudiantes. Pero no hemos evaluado si los estudiantes han aprendido y cuánto. Por eso, se trata de una evaluación indirecta de la calidad. Para tener una evaluación directa, última y genuinamente auténtica de la calidad de la educación, tenemos que saber si los estudiantes que han estudiado en esa Universidad poseen un nivel elevado de conocimientos y, más aún, si son capaces de aplicar esos conocimientos para tomar decisiones y resolver problemas efectivamente en la realidad donde se van a desempeñar como profesionales. Pero, la evaluación directa y real de la calidad de la educación es muy difícil y costosa de realizar. Por esta razón, se evalúa indirectamente, haciendo inferencias sobre la calidad de la educación que puede ofrecer una organización educativa que posea elevados valores en los estándares de calidad utilizados para evaluar sus características estructurales y funcionales consideradas ideales.

Los estudiosos de la calidad de la educación virtual a distancia coinciden en que ella posee una especificidad propia y su evaluación no puede reducirse a los mismos criterios que la educación no-virtual presencial. Es posible que existan criterios aplicables por igual a ambas modalidades educativas, pero al adentrarnos en su análisis encontraremos especificidades de la educación virtual a distancia que requiere de criterios y metodologías particulares para evaluar su calidad. No obstante, mi opinión sobre este tópico consiste en partir del principio de la unicidad de la educación. La educación es una sola pero puede adoptar diversas modalidades combinadas, para ofrecer oportunidades equivalentes a distintos tipos de actores 
de manera flexible. Hay que tener presente la unicidad, flexibilidad y equivalencia de la educación al evaluar su calidad.

El interés por la educación virtual a distancia ha ido acompañado de una proliferación de concepciones sobre la evaluación y gestión de su calidad. En muchos países en desarrollo la preocupación por la calidad ha conducido al desarrollo de sistemas nacionales de evaluación y acreditación de la educación superior, que existían ya en países desarrollados desde hace un cierto tiempo, pero que en el mundo en desarrollo son una novedad muy reciente. El objetivo es desarrollar mecanismos de evaluación y control de la calidad de la educación superior y monitorear de manera permanente el desempeño de las instituciones de educación superior. En este sentido, el proyecto del CREAD consistente en desarrollar un sistema de estándares de calidad de la educación en América Latina, con el apoyo de varias instituciones y universidades de esta región y de Estados Unidos y Europa, es una iniciativa que viene a llenar una necesidad largamente sentida en América Latina. El objetivo del programa es el desarrollo de las bases para un sistema de estándares de calidad para la evaluación y acreditación de programas de educación a distancia en América Latina y el Caribe (CREAD-UTPL, 2004: 93-94)

\section{Gestión}

La evaluación de la calidad y la acreditación resultante de ella es sólo un aspecto del problema. Lo importante después de eso es asegurar, mantener y mejorar continuamente la calidad, lo cual requiere de una buena gestión. La evaluación proporciona los elementos necesarios para monitorear permanentemente el desarrollo de los programas educativos y su mejoramiento, al saber cuánto y cómo se han desviado de sus objetivos y de las necesidades de los usuarios. Pero sólo la gestión garantiza ese monitoreo permanente.

La gestión consiste en planificar, conducir, monitorear, evaluar y controlar un conjunto interdependiente de actividades y tareas para la toma decisiones y la solución de problemas con miras a lograr determinados objetivos. En nuestro caso, lograr los objetivos relacionados con mantener y mejorar la calidad de un sistema educativo a distancia.

La educación a distancia actual está muy influenciada por las tecnologías digitales de información y comunicación o lo que hemos llamado tecnología instruccional. Otra definición más relevante para los propósitos que perseguimos consiste en relacionar la gestión, la calidad y la tecnología instruccional en el marco de la educación virtual y a distancia. Esa definición podría enunciarse de la siguiente manera:

Es el proceso de planeamiento, conducción, monitoreo, evaluación y control de actividades y tareas para la toma de decisiones y solución de problemas sobre la teoría y práctica del diseño, desarrollo, la gestión, la utilización y la evaluación de 
procesos y recursos para el mantenimiento y mejoramiento de la calidad del aprendizaje virtual y a distancia.

Esta definición es relativamente compleja, pero comprende los componentes principales de la gestión de la calidad de la tecnología instruccional aplicada a la educación virtual y a distancia. Si la descomponemos en sus elementos principales obtenemos los componentes que necesitamos para integrar un sistema coherente y efectivo para la gestión de la calidad de la educación virtual y a distancia.

\section{Liderazgo}

El proyecto del CREAD sobre estándares de calidad en la educación a distancia comprende un criterio especialmente relevante para los objetivos de este trabajo, perteneciente al grupo de criterios sobre Procesos Facilitadores. El Criterio 1 se refiere al Liderazgo y Estilo de Gestión La evaluación de la calidad de un programa educativo según este criterio requiere estudiar cómo se desarrolla y aplica la estructura organizacional del programa, el marco de los procesos y su sistema de gestión, necesarios para la eficaz ejecución de la Política y la Estrategia de la Institución, mediante adecuados comportamientos y acciones de los responsables del programa, actuando como líderes (CREAD-UTPL, 2004). El liderazgo y la gestión derivada de sus características son los instrumentos ejecutores de la política y estrategia de la organización educativa objeto de evaluación, para el logro de una buena calidad de la educación distancia (CREAD-UTPL, 2004).

El liderazgo es un componente indisociable de la gestión y de la sociabilidad humana en general. Basta que se le asigne una tarea a un grupo de personas para que surja una estratificación sobre la base de las habilidades de cada una. Con el tiempo, surgirá una persona líder (además de otras que puedan surgir con otras habilidades) con una mayor capacidad para manejar las personas y lograr que orienten su conducta en un sentido deseado. El liderazgo es, entonces, la capacidad de una persona, grupo, organización, comunidad y sociedad para orientar la conducta de los demás en un sentido deseado. El liderazgo puede ser entonces ejercido por actores individuales y colectivos, una persona con poder político, un grupo político, una empresa, una Universidad. Igualmente, puede abarcar diversos niveles y distintas áreas institucionales de manifestación de la conducta humana en la sociedad.

Además de distinguir el liderazgo formal del informal, se puede ejercer liderazgo en las acciones, los pensamientos y sentimientos de la gente. Un líder puede lograr que la gente actúe, piense y sienta lo que desea y como él lo desea. El liderazgo comprende estas tres dimensiones: acción, pensamiento y sentimiento. La tridimensionalidad del liderazgo se puede ilustrar mediante un diagrama como el de la Figura 2, en cual se pueden representar los valores de cada dimensión y de sus combinaciones. 


\begin{tabular}{|l|l|l|l|}
\hline & Acciones & \multicolumn{1}{|c|}{ Pensamientos } & Sentimientos \\
\hline Acciones & $\begin{array}{l}\text { Liderazgo } \\
\text { de Acción }\end{array}$ & & \\
\hline Pensamientos & & $\begin{array}{l}\text { Liderazgo Intelectual o de } \\
\text { Opinión }\end{array}$ & \\
\hline Sentimientos & & & Liderazgo Espiritual \\
\hline
\end{tabular}

Figura 2. El liderazgo y sus relaciones con las acciones, los pensamientos y los sentimientos

Los tres tipos básicos puros de liderazgo son el líder de acción (influye sobre las acciones solamente), el líder intelectual o de opinión (influye sobre los pensamientos solamente) y el líder espiritual (influye sobre los sentimientos solamente). Podemos encontrar combinaciones de uno con los otros dos tipos de liderazgo partiendo de la ausencia de combinación (sólo los tipos puros) hasta la combinación de los tres tipos en uno que podríamos llamar liderazgo integral o liderazgo de acción, pensamiento y sentimiento.

Es interesante establecer una relación entre un paradigma y el liderazgo. Un paradigma es un conjunto de reglas interdependientes que nos sirven para concebir un aspecto del mundo. El paradigma define la manera como pensamos, actuamos y sentimos sobre un área de sociabilidad del mundo. Si un líder puede influir sobre la manera como actuamos, pensamos y sentimos, entonces es portador de un determinado paradigma de acción, pensamiento y sentimiento y es un instrumento de gestión de ese paradigma. La educación a distancia es portadora de un paradigma distinto que comprende otra manera de pensar, actuar y sentir sobre la educación. El conocimiento de ese nuevo paradigma educativo y la manera de ejercer un liderazgo efectivo para implantarlo en la sociedad y vigilar y mejorar su calidad, son tareas prioritarias para quienes deseen desarrollar la educación a distancia.

\section{Innovaciones y su difusión en la sociedad}

Una innovación es algo considerado diferente y nunca visto en comparación con lo que existe en el ambiente de una persona, grupo, organización o sociedad. Puede ser un objeto material (automóvil, artefacto electrónico, un libro, etc.) o inmaterial (una idea, un servicio, una técnica, una organización, etc.) Su novedad depende del grado en que el objeto sea percibido como nuevo y distinto a lo que ya existe para una persona, grupo, organización y sociedad. En conclusión, hay una dimensión objetiva y otra subjetiva de la innovación y varios niveles de manifestación y difusión de las innovaciones (persona, grupo, organización y sociedad). Estas dimensiones y estos niveles, permiten determinar la intensidad o profundidad de una innovación, su grado de difusión en la sociedad y la diversidad de ambientes en los cuales se ha difundido. 
La educación a distancia puede ser considerada como una innovación. Si bien tiene ya un cierto tiempo de existencia, el uso de tecnologías digitales de información y comunicación y la aparición de la virtualidad le han dado un impulso muy importante, que le ha permitido adoptar una nueva identidad y un papel significativo de mayor credibilidad en la sociedad. Lo nuevo en la educación a distancia es la virtualidad, la nueva tecnología instruccional y los medios tecnológicos digitales informáticos y telemáticos que le sirven de soporte y de instrumento. En efecto, el desarrollo de la informática y la telemática ha brindado una nueva forma de comunicación y de sociabilidad en un espacio totalmente nuevo, el ciberespacio. Hay, pues, mucha novedad en la manera como ahora podemos informarnos y comunicarnos para enseñar y aprender, que no existía cuando surgió la primera edición de la educación a distancia. Es una innovación de un alto grado de intensidad y de variedad de situaciones. Por esta razón, la gestión de la calidad de la educación a distancia es ahora y seguirá siendo en el futuro un desafío para todos los actores que en ella participan de una u otra manera y desempeñando diversos roles. El ejercicio de un liderazgo efectivo para asegurar una gestión adecuada de la calidad de la educación a distancia es, por eso, una tarea que debe realizarse de manera cuidadosa y sistemática. Estamos realizando la gestión de la difusión de una innovación que implica cambios intensos y profundos en las prácticas de la enseñanza y del aprendizaje. Entonces, en este contexto, es muy relevante y significativo estudiar las características de un proceso de difusión de innovaciones.

Everett Rogers (2003) ha dedicado toda su vida a estudiar las innovaciones y la manera como se difunden en la sociedad y es reconocido como uno de los especialistas más destacados en este campo. La teoría de la difusión de innovaciones se propone explicar la manera como las personas, los grupos, las organizaciones, comunidades y sociedades adoptan una innovación. La difusión es el proceso a través del cual una innovación es comunicada mediante ciertos canales durante un tiempo específico entre los miembros de un sistema social. En consecuencia, los cuatro elementos principales de la teoría son:

- la innovación,

- los canales de comunicación,

- $\quad$ el tiempo y

- el sistema social.

La combinación de los valores y situaciones de estas variables ofrece las variantes particulares y específicas de cada proceso de difusión de una innovación.

El proceso de difusión sigue una curva en forma de S como se muestra en la Figura 3 y en ella se representa la velocidad de adopción y el número de adoptantes. Al principio, la innovación se difunde lentamente, pero a medida que la innovación se va adoptando por un número cada vez mayor de actores, comienza a difundirse de manera más rápida. Luego, el número de adoptantes disminuye al llegar a un punto de saturación y comienza a estabilizarse. 


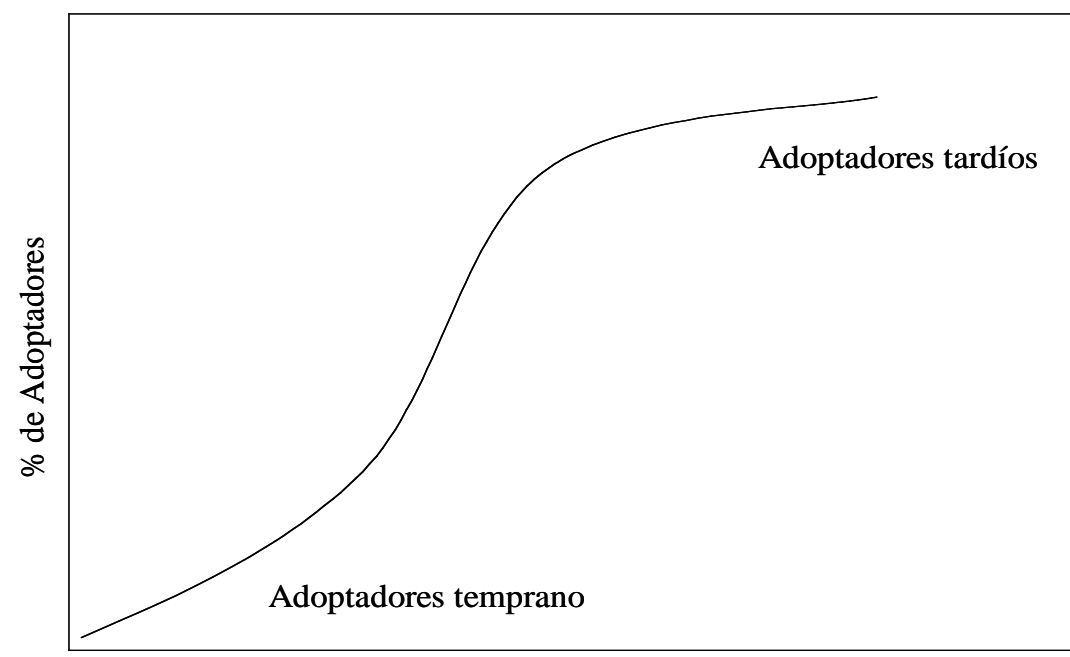

Tiempo

Figura 3. Curva de Adopción de Innovaciones según Rogers

Fuente. Rogers, Everett (2003) Diffusion of innovations. 5a. Edición. Free Press.

New York.

Existen, según Rogers, cinco etapas en el proceso de difusión:

1. Conocimiento: la persona adquiere un primer conocimiento sobre la innovación.

2. Comunicación interés o persuasión: la persona comienza a comunicarse con otras en relación con la innovación, comienza a formarse actitudes sobre la innovación.

3. Decisión: la persona busca información para disminuir la incertidumbre en relación con la innovación, para así poder tomar una decisión sobre su adopción.

4. Prueba o implementación: consiste en usar regularmente la innovación, para obtener más información sobre sus ventajas y desventajas;

5. Confirmación: la persona ya es usuaria regular de la innovación y busca confirmar si ha tomado una decisión correcta.

Después de la etapa de confirmación, puede decirse que la innovación se ha enraizado en la sociedad y las personas la han aceptado e internalizado como parte de sus patrones de comportamiento usuales.

La curva de adopción de innovaciones permite también clasificar a los adoptantes de una innovación en varias categorías, sobre la base de la idea según la cual algunas personas son más abiertas que otras a la adopción que otras. 
Rogers clasifica a los actores o personas involucrados en cinco grupos, en relación con este aspecto:

- Los innovadores: son quienes asumen los riesgos de introducir y difundir la innovación. Generalmente, son los propios productores de la innovación, acompañados de un grupo de personas usuarios entusiastas, que pueden ejercer influencia sobre otras personas.

- Adoptadores tempranos: son quienes adoptan la innovación por primera vez y sin mucha discusión y análisis; pueden actuar como líderes reconocidos y respetados y jugar un papel importante como líderes de influencia para persuadir a otros actores de adoptar la innovación.

- La mayoría temprana: comprende los actores que no están dispuestos a correr riesgos de diversa índole y arriesgar tiempo y otros recursos, tienen cierta resistencia al cambio, analizan y reflexionan cuidadosamente antes de tomar una decisión; sin embargo, son propensos a aceptar la innovación después de una actividad persuasiva relativamente corta.

- La mayoría tardía: son sumamente resistentes a cambiar, son difíciles de adoptar la innovación sin una actividad persuasiva intensa y una influencia significativa.

- Rezagados: es la categoría de personas más refractarias al cambio, se muestran indiferentes ante cualquier innovación e incluso llegan a oponerse a ella y a combatirla activamente; son celosos guardianes del status quo y con frecuencia nunca llegan a adoptar la innovación.

De acuerdo a los estudios realizados por Rogers, estas categorías de personas se distribuyen siguiendo una curva normal, como se muestra en la Figura 4. Primero, encontramos una minoría muy pequeña de innovadores (2,5\%) acompañada de un conjunto de adoptadores tempranos (13,5\%). A estos se agrega la mayoría temprana, que reúne aproximadamente $34 \%$. Al final encontramos una mayoría tardía (34\%) y un pequeño conglomerado de rezagados (16\%).

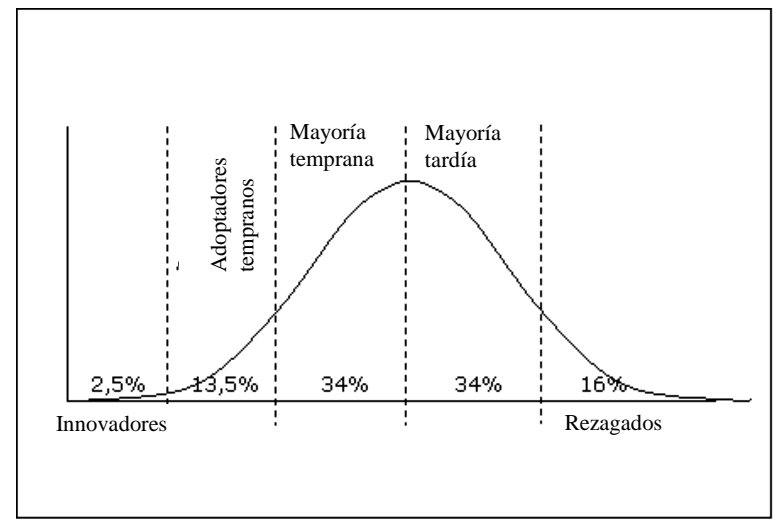

Figura 4. Categorías de adoptadores de una innovación y porcentaje de la población total de adoptadores Fuente: Rogers, Everett (2003) Diffusion of innovations. 5a. Edición. Free Press. New York. 
Hay algunas características de la innovación que ayudan a determinar su éxito, a saber:

- La complejidad de la innovación, que consiste en el grado de dificultad que los actores, como usuarios potenciales, experimentan para comprender las características y el uso de la innovación.

- La compatibilidad de la innovación con los valores y pautas de conducta del grupo social receptor, de sus necesidades y en relación con otros objetos materiales o inmateriales, que ella sustituye o complementa.

- La ventaja relativa, es decir, el grado en el cual una innovación es percibida como mejor que lo que ella está llamada a sustituir.

- La disponibilidad para probar y utilizar la innovación es otra condición importante a considerar.

- La observabilidad se refiere al grado en el cual la innovación ofrece resultados visibles o manifiestos para el usuario.

Además de estas características, antes de intentar introducir una innovación en cualquier área institucional y organizacional de un sistema social, es necesario tomar en cuenta los siguientes aspectos:

- Las características de la población objetivo, es decir, la población receptora y usuaria potencial, sus categorías estimadas de adoptantes y el proceso típico de toma decisiones para adoptar la innovación.

- Las características de la innovación que la hacen susceptible de ser adoptada por la población.

- Las consecuencias para las personas, grupos, organizaciones y sociedades de adoptar la innovación.

- Los canales de comunicación utilizados para difundir la innovación.

- Las etapas de adopción estimadas.

Es deseable analizar cada aspecto y planificar la introducción y el desarrollo de la innovación en función de sus resultados.

La importancia de la teoría de la difusión de las innovaciones reside en que su conocimiento es importante para poder planificar, conducir, monitorear, evaluar y controlar de manera racional y sistemática cualquier decisión que se tome o acción que se realice para introducir y desarrollar cualquier innovación como la educación virtual y a distancia.

A este nivel del desarrollo de este trabajo, el lector se preguntará ¿cuál es la relevancia y significación de todos estos conceptos y teorías expuestos hasta ahora para el ejercicio del liderazgo en la gestión de la calidad de la educación virtual y a distancia? La respuesta a esta pregunta será el objeto del resto de este trabajo. 


\section{GESTIÓN DE LA CALIDAD EN LA DIFUSIÓN DE LA EDUCACIÓN A DISTANCIA COMO INNOVACIÓN}

Vamos ahora a integrar los conceptos definidos para formular algunas propuestas con el propósito de desarrollar el liderazgo en la gestión de la calidad de la educación a distancia. Hay tres ideas directrices principales en esta integración. Primero, la idea según la cual la tecnología instruccional digital es la base de la educación a distancia actual y la gestión de su calidad dependerá mucho de la gestión de esa tecnología. Segundo, la idea del liderazgo como motor de la gestión en general y de la gestión de la calidad de la educación a distancia, en particular. Tercero, la idea de que la educación a distancia es una innovación y es posible gestionar su difusión en la sociedad de manera sistemática.

La tarea que tendrán los líderes de la educación a distancia es introducir y difundir la educación distancia en la sociedad. Pero la tarea no termina allí sino que deberán procurar lograr una alta calidad de la educación a distancia, difundirla y luego mantener esa calidad y mejorarla en el tiempo. Los líderes, con la ayuda del personal técnico necesario, deberán primero evaluar las condiciones en las cuales se va a introducir, a saber:

- las características de la población objetivo;

- las características de la innovación y

- las etapas de adopción estimadas.

Los líderes de los programas de educación a distancia y su núcleo inicial actúan como innovadores, un pequeño grupo que según Rogers representa aproximadamente 2,5\% de la población. Ellos también son usuarios de la innovación, pero su función es persuadir y convencer a la población de usar la innovación.

La primera población que deben persuadir y convencer los innovadores es la población que sería la de los adoptadores tempranos, la cual puede considerarse también como una minoría temprana. Estos son usuarios permeables a las innovaciones, que pueden ser persuadidos con relativa facilidad y convertirse en líderes de opinión, capaces de influir sobre otras poblaciones. Esta población de adoptadores tempranos está lejos de ser homogénea. Incluye actores que ocupan distintas posiciones y desempeñen diferentes papeles en el sistema educativo. Por ejemplo, allí encontraremos profesores, estudiantes, administradores y altas autoridades educativas y también líderes de los medios de comunicación social masivos. Cada categoría social de actores requiere de una estrategia diferente de difusión de la innovación, en virtud de diversas variables, su status, rol, sus valores, necesidades, expectativas y aspiraciones. Es preciso tener presente que los actores y usuarios más importantes son los estudiantes. Deseo enfatizar esto, pues con mucha frecuencia se olvida y se le da más importancia a la maquinaria que se estructura y pone en movimiento para producir aprendizaje (el sistema educativo), que a los usuarios y beneficiarios directos de la misma: los estudiantes. Los estudiantes y la calidad del aprendizaje que han realizado y de los conocimientos que han adquirido, son la 
medida última y definitiva de la calidad de la educación a distancia. Son, para usar un lenguaje más propio del mercadeo, los clientes más importantes de los sistemas de educación a distancia y a quienes se necesita convencer de que usen el sistema.

Para convencer a los adoptadores tempranos, la situación se presenta relativamente fácil. Es una población propensa a las innovaciones, que fácilmente va a aceptar la educación a distancia y transitará rápidamente por las etapas de la innovación: conocimiento, persuasión, decisión, implementación y confirmación. Muchos de los adoptadores tempranos serán usuarios con cierto grado de propensión al uso de la innovación. Se requiere dirigir los esfuerzos hacia usuarios que tengan algún conocimiento sobe el uso de la informática y la telemática y hayan tenido ya una experiencia de educación a distancia apoyada por la tecnología digital, sea como usuario final o estudiante o como profesor o administrador o directivo. Es necesario procurar manejar a esta población con mucho cuidado, pues dada la influencia que pueden tener sobre las decisiones y acciones de otras poblaciones, pueden determinar buena parte del éxito de la difusión de la educación a distancia. Si esta población pone trabas y no queda del todo convencida, puede que el programa de educación a distancia pase la prueba, pero sin una gran convicción y con poca proyección hacia otras etapas y otras poblaciones. Generalmente, a esta población se le puede hacer transitar más rápidamente las etapas de introducción y difusión de la innovación haciéndolos participar en una prueba o experiencia piloto del programa de educación a distancia a introducir. Además de ofrecer pruebas convincentes del uso y la utilidad de la educación a distancia para el usuario, es necesario ganarse esa población como aliados para ejercer un cierto liderazgo en el convencimiento y la persuasión de otras personas pertenecientes a otras categorías de usuarios.

En esta categoría de actores es necesario poder identificar quienes podrían ayudar a los innovadores a llegar a la mayoría temprana de usuarios. Una vez consolidada esta población de usuarios, debemos pasar a trabajar con la próxima población, la población de la mayoría temprana.

La mayoría temprana se compone de personas que no son propensas a utilizar la educación a distancia como los adoptadores tempranos. Generalmente se trata de personas que podrían adoptar la educación a distancia como modalidad educativa, pero necesitan un trabajo de conocimiento y persuasión mayor que los adoptadores tempranos. Es necesario recurrir en esta etapa a las redes sociales de difusión de innovaciones y a la manera como en ellas operan los líderes y su influencia (Valente, 1995). Cada persona tiene su red personal y la moviliza cuando desea realizar una acción de su interés en su red personal, la cual varía según el rol que desempeña la persona en cada área de conducta (Rogers, 1980: 114-153). La relevancia de considerar estas redes consiste en que son instrumentos muy importantes para que los innovadores, apoyados por los adoptadores tempranos y el liderazgo que estos últimos pueden ejercer a través de sus redes sociales, puedan introducir con fluidez la educación a distancia en la mayoría temprana y lograr su adopción como innovación. 
En esta mayoría temprana encontramos personas de muy variado origen, diversos status y diferentes roles. Es una población mucho más numerosa y diversificada que las anteriores. Todo ello sugiere la conveniencia de segmentar esta población, para identificar mejor las redes sociales de difusión de la educación a distancia que pudieran existir y sean utilizables como recursos para difundir la educación a distancia.

La mayoría temprana será más difícil de persuadir que los adoptadores tempranos quienes, por definición, son propensos a aceptar la educación a distancia. A esta población habrá que convencerla mostrándole la ventaja relativa de la educación a distancia respecto a otras modalidades educativas: ¿por qué estudiar y/o enseñar a distancia en vez de mediante otras modalidades? ¿Es de mejor calidad la modalidad educativa a distancia en comparación con lo que existe? En este aspecto, conviene recordar lo referente a la articulación de modalidades educativas sobre la base del espacio, el tiempo y la virtualidad. No debemos ofrecer a los usuarios potenciales la alternativa de escoger entre educación virtual y a distancia o la educación presencial y no-virtual. La innovación a difundir y su calidad a mantener no consiste en adoptar una en sustitución de la otra, sino articular la innovación con las características más relevantes y compatibles con lo que existe. Igualmente, debemos asegurar su compatibilidad con los patrones culturales y los valores de la población usuaria. Este es otro criterio que permitirá apreciar más fácilmente la calidad de la innovación a difundir. De esta manera, el usuario de la mayoría temprana puede integrar la innovación dentro de mapas mentales familiares y no percibir que se está dando una especie de salto al vacío o hacia un ambiente completamente desconocido, en el cual el usuario estará relativamente perdido y será incapaz de manejarse adecuadamente en ese ambiente.

La educación a distancia, o el programa respectivo, debe presentarse con el menor grado de complejidad posible, a fin de que los usuarios puedan percibir la calidad de la innovación que se les pide adoptar y utilizar. Ello se podrá lograr si podemos alcanzar el mayor grado de empatía en la comunicación con los usuarios de la mayoría temprana, que consiste en tratar de ponernos en su lugar y tratar de pensar, actuar y sentir como ellos. Para que todo esto sea posible, la innovación a introducir y difundir debe tener disponibilidad para que los usuarios puedan probarla y utilizarla y procurar que los resultados obtenidos después de utilizarla tengan la mayor observabilidad, es decir, se puedan observar claramente sus resultados de forma manifiesta y comprobar su calidad en relación con otras modalidades educativas.

El primer punto de consolidación de la introducción y difusión de la educación a distancia como innovación, lo constituyen, sin duda, los innovadores, que son los actores introductores. Su primer punto de apoyo son los adoptadores tempranos y la consolidación debe venir a partir de las redes sociales en las cuales participan estos actores y el liderazgo que pueden ejercer en ellas. A nivel de los adoptadores tempranos, se corre el riesgo de que la educación a distancia quede encapsulada como innovación, utilizada sólo por los innovadores y un número relativamente pequeño de entusiastas adoptadores tempranos. Esto es lo que muchas veces ha 
ocurrido, si no se toman en cuenta las condiciones y características ya señaladas: ventaja relativa, compatibilidad, facilidad de uso, disponibilidad y observabilidad. Muchas innovaciones se quedan en la cápsula, aún mostrando un alto grado de calidad, capaces de resolver importantes problemas a los usuarios y muy portadoras de futuro, porque sus actores difusores no han podido penetrar en la mayoría temprana.

La mayoría temprana es tal vez la población más importante porque es el punto de inflexión de la curva de adopción de innovaciones identificada por Rogers y sus colaboradores. A partir de allí es como si se traspasara a otro umbral (el primero es el de los adoptadores tempranos) que permite llegar a la consolidación de la innovación.

La población de innovadores, adoptadores tempranos y mayoría temprana constituyen ya una masa crítica que representa $50 \%$ de la población total de usuarios. Además, esa masa crítica se caracterizará por el hecho de que serán usuarios convencidos, con un cierto nivel de experticia en la utilización de la educación a distancia y capaces de ejercer liderazgos de intensidad y tipo variables sobre los usuarios vinculados a sus redes personales de influencia. La identificación de los líderes de la mayoría temprana es tan importante como la de los adoptadores temprano. Ellos podrán ejercer un liderazgo, aunque tal vez no de una manera tan efectiva y comprometida como lo puede hacer un actor adoptador temprano a quién se le haya ganado como adepto a la causa de la innovación, en este caso, la educación a distancia. Pero, pueden ejercer el liderazgo de un usuario convencido de la utilidad y calidad de la educación a distancia y capaz de evaluarla de manera más objetiva que otros actores más imbuidos por la innovación.

La introducción y difusión de la educación a distancia y la vigilancia del mantenimiento de su calidad y su mejoramiento, es una tarea de ejercicio de un liderazgo racional pero sin dejar de lado los aspectos emocionales. Es también un trabajo en equipo de varios líderes de un mismo segmento de población y de otros segmentos, al igual que otros actores que no ejerzan liderazgo pero cuyas acciones, pensamientos y sentimientos puedan influir sobre las decisiones de los demás en relación con la educación a distancia.

Luego de consolidar la innovación en estas poblaciones, hay que pasar a la siguiente población, la mayoría tardía, que representa más o menos $34 \%$ de la población. Se trata de una población de gente escéptica, pero que no se opone por principio a la educación a distancia. Además de esta ventaja relativa, hay otra que consiste en ser una población que usaría la educación a distancia una vez que compruebe que la mayoría temprana la ha adoptado ya y la reconozca como un producto de buena calidad, confiable. Esta población no asume muchos riesgos, prefiere que los asuman primero otros de la mayoría temprana que son más propensos a correrlos. Son personas influenciables más por la tradición y utilizarán la educación a distancia cuando la mayoría temprana la haya adoptado en su mayor parte. Ellos necesitan pruebas inequívocas de la calidad y utilidad de la educación a distancia. La mayoría temprana es una importante categoría poblacional de referencia para la 
mayoría tardía. Así, es muy importante formar líderes de opinión en la mayoría temprana que puedan darle a la mayoría tardía la seguridad y confianza que necesita para adoptar y utilizar la educación a distancia

Con la mayoría tardía tenemos entonces una adopción de 84\% de la población usuaria. El 16\% restante corresponde a la población más difícil: los rezagados. Esta categoría engloba personas refractarias y opuestas a la innovación, que puede llegar incluso a ser irracional y a oponerse a la educación a distancia simplemente por tratarse de algo nuevo, distinto a lo conocido y lo existente. Adoptarían la educación a distancia en un plazo muy largo, cuando ella se convierta casi en una tradición. Estaríamos tentados a ignorar esta población, debido al bajo porcentaje de personas que agrupa, pero no es aconsejable. Es deseable tener un conocimiento cabal de sus características y de las posiciones ocupadas por ellas en la sociedad.

En esa categoría de población pueden hallarse personas que ocupen posiciones de liderazgo importantes en la sociedad y ellas podrían influir negativamente en la introducción y difusión posterior de la educación a distancia. Puede ser muy dañino para un proceso de innovación el poder que podría ejercer un rezagado que posea un liderazgo de influencia y decisión sobre la población. Muchos proyectos innovadores han quedado inactivos por el bloqueo impuesto por personas y grupos de actitudes de rezago y rechazo. Con estas personas hay que proceder con cuidado y evitar que su rezago se convierta en un rechazo activo, es decir, un rechazo que conduce a los actores a actuar para oponerse a la innovación y bloquear su difusión en la sociedad.

En consecuencia, es necesario que los innovadores, con la ayuda de los adoptadores temprano logren identificar a esta población de rezagados y a sus líderes, desde el principio o en las primeras fases de gestión de la difusión de la innovación. Sería ideal que se pudieran identificar y actuar sobre ellos antes de difundir la innovación en la mayoría temprana. Si los rezagados logran perturbar o detener la difusión antes de que llegue a esta población, se corre el riesgo de que quede encapsulada y restringida al pequeño número de innovadores y de adoptadores tempranos. No se trata de promover y administrar un conflicto entre innovadores y rezagados. Se trata de identificar en la población rezagada sus expectativas y temores. En este contexto, conviene hacer un análisis de oportunidades, amenazas, fortalezas y debilidades, lo cual es aplicable a otras poblaciones. Con respecto a la población rezagada, es de especial interés identificar y analizar las amenazas que perciben en la educación a distancia y dilucidar de qué manera se pueden convertir en oportunidades de desarrollo. Con esta óptica se puede negociar mejor con esos actores propensos a oponerse a la innovación. El objetivo más deseable al ejercer un liderazgo para realizar una gestión efectiva de la difusión de la educación a distancia y mantener y mejorar su calidad, es lograr que ella sea adoptada por el mayor número de personas. Para ello, hay que tratar de lograr que todos puedan utilizar la educación a distancia de acuerdo a sus posibilidades, necesidades, expectativas y aspiraciones. Una estrategia conducida por el objetivo de ganar-ganar. Para lograr este objetivo, se requiere, en primer lugar, partir de la premisa de la unicidad de la educación, es decir, la educación es una sola pero adopta varias modalidades. En 
segundo lugar, para satisfacer las necesidades, expectativas y aspiraciones de todas las poblaciones susceptibles de aprender y enseñar de acuerdo a esas modalidades, se requiere garantizar al usuario un alto grado de flexibilidad y facilitar la articulación adecuada entre la educación a distancia y presencial y virtual y no-virtual. Al mismo tiempo, se debe procurar que las experiencias de aprendizaje ofrecidas a los actores se guíen por el principio de equivalencia. De acuerdo a este principio, dos experiencias de aprendizaje pueden ser diferentes pero es deseable sus resultados en términos de cantidad, tipo y calidad del aprendizaje obtenido por los usuarios.

Es deseable y necesario procurar que cada usuario encuentre en un programa articulado de educación a distancia su mejor mundo entre dos mundos y pueda aprender y enseñar de manera flexible e integrar el aprendizaje y la enseñanza de manera natural (Schlosser y Burmeister, 1999). Un programa de educación a distancia que se proponga responder a las necesidades de aprendizaje y a sus expectativas y aspiraciones de distintos grupos sociales, deberá tener la suficiente flexibilidad para que los diferentes grupos de usuarios puedan adaptar el programa lo mejor posible a sus necesidades, expectativas y aspiraciones.

La calidad adquiere nuevas dimensiones con la articulación y las características asociadas a ella, requiere una gestión muy especial y un liderazgo particular sobre todo para conducir el proceso, difundir significativamente la innovación, mantener su calidad y mejorarla continuamente. Igualmente, es necesario considerar la cadena de valor particular que se establece cuando se realiza la gestión de un programa de esa naturaleza. Hay muchos actores involucrados, de diversas poblaciones, sumiendo distintos roles. De la conducta de cada actor, en cada una de las etapas del proceso de difusión, dependerá en gran medida, no sólo el éxito de la difusión de la educación a distancia, sino, al mismo tiempo, el mantenimiento y mejoramiento de su calidad.

Un programa educativo con las características señaladas y que se guíe por los principios de unicidad de la educación, articulación, flexibilidad y equivalencia, adquiere una complejidad que no encontramos en otros programas. Se trata de un programa en continuo movimiento en equilibrio dinámico, que necesita ajustarse permanentemente. En este caso, toda la estructura y la funcionalidad del sistema de gestión de la calidad y del liderazgo requerido deben también funcionar en estado de equilibrio dinámico. De esta manera, la educación a distancia podrá difundirse con más fluidez en las diferentes categorías de poblaciones adoptantes de la innovación. Sólo con flexibilidad, articulación y equivalencia se puede producir una mayor diversidad de oportunidades de aprendizaje a distancia utilizables por todas las categorías de población adoptante. Así, podrían agregarse nuevos criterios de evaluación indirecta de la calidad de la educación a distancia, a saber: el grado y tipo de articulación, la flexibilidad y la equivalencia de un programa determinado, que se podría medir sobre la base de indicadores a construir especialmente con esa finalidad. Mientras más articulación entre lo virtual y lo no-virtual y lo presencial y lo distante, más flexibilidad tenga un programa educativo a distancia y más equivalentes sean las diversas experiencias de aprendizaje ofrecidas a los usuarios, su ca- 
lidad será mayor y podrá satisfacer a usuarios de diversas poblaciones de usuarios y con distintas necesidades, expectativas y aspiraciones en materia de aprendizaje.

La calidad tendrá un significado diferente y requerirá una manera distinta de evaluarla según el usuario y la variante de oferta educativa que le sea presentada. La calidad del programa residirá en su capacidad para ofrecer variedad, flexibilidad, articulación y experiencias equivalentes de aprendizaje a sus usuarios.

Sin duda que un programa educativo diversificado y capaz de satisfacer a diversos tipos de usuarios, requiere de un liderazgo y un gestión especialmente dinámicas y adaptativas y más aún proactivas, es decir un liderazgo y una gestión que realicen acciones hacia delante, en las cuales no se reaccione ante lo que ocurre sino que anticipe lo que pueda ocurrir y actúe en consecuencia.

El mayor grado de calidad en la educación a distancia se alcanzará cuando ella se pueda difundir y administrar como innovación de una manera natural y relativamente invisible. La metáfora de la invisibilidad para ilustrar el grado en que una innovación se integra con nuestra vida ha sido ya utilizada en el mundo de la computación. Begoña Gros (2000) ha escrito un libro titulado El Ordenador Invisible, en el cual plantea que el ordenador pasará a integrarse a la educación cuando nos apropiemos de él y de la tecnología de la cual es portador y ya no se hable de él, cuando sea algo invisible, cuando al entrar en el aula los ordenadores nos parezcan tan naturales como los bolígrafos, los libros o los cuadernos (Gros, 2000). Por su parte, Mark Weiser vislumbró la llegada y difusión en la sociedad de lo que llamó computación ubicua (ubiquitous computing) o también la computación de la ubicuidad. Según Weiser, en esa nueva era, que ya ha comenzado, la tecnología y los medios tecnológicos físicos que la acompañan y la hacen realidad (computadoras y otros artefactos) se integrarán progresivamente a nuestra vida cotidiana, no como algo externo sino como parte de nuestro entorno y de nuestros paradigmas de pensamiento, acción y sentimiento. Si la computación pudiera tener las características de la ubicuidad, impregnando todos los aspectos de nuestra vida y sus artefactos lleguen a ser como una extensión natural de nosotros, se hará entonces invisible. Por eso, Weiser decía que "...las buenas tecnologías son las que se desvanecen y se funden de tal forma con nuestra vida cotidiana, que se vuelven invisibles." (Weiser, 1996).

El liderazgo más efectivo para garantizar y mejorar continuamente la calidad de la educación a distancia y la tecnología instruccional que la acompaña y su difusión como innovación en la sociedad, es aquel que se basa en el conocimiento de las variables, conceptos y principios expuestos y sabe combinarlas adecuadamente en una estrategia efectiva para el logro de ese objetivo. Debe ser un liderazgo participativo y distributivo al mismo tiempo. Participativo porque debe facilitar la participación de todos en los procesos de toma de decisiones y la realización de acciones. Distributivo porque el poder y la autoridad en este complejo proceso debe distribuirse adecuadamente entre todos los actores involucrados en la gestión.

La gestión de la calidad en la educación a distancia debe realizarse independientemente de que exista un sistema formal de evaluación y control de la calidad. De 
no existir tal sistema estructurado, los actores que intervienen en el proceso deben llegar a acuerdos y establecer unas normas mínimas aceptadas para aplicarlas a la evaluación y gestión de la calidad. Aunque no exista nada y el docente se vea en la situación de tener que trabajar solo, él debe convertirse en gerente y realizar la implementación y la gestión de la calidad al mismo tiempo. La gestión de la calidad debe efectuarse en cualquier situación. Todos somos gerentes, desde el momento en que planificamos, conducimos, evaluamos y controlamos un conjunto de actividades para tomar decisiones y resolver problemas para lograr determinados objetivos de enseñanza y aprendizaje, en el marco situacional de la educación virtual y a distancia. Todo docente es líder y gerente de la calidad, al mismo tiempo. Como tal, debe estar adecuadamente capacitado para ser líder y gerente, aunque tenga que trabajar solo y sin un marco estructurado para evaluar y gerenciar la calidad. La gestión de la calidad debe comenzar a nivel micro, en el aula virtual con los actores principales (profesores y estudiantes). Igualmente, debe formarse adecuadamente a los estudiantes para que vigilen la calidad de lo que están aprendiendo.

Como hemos visto, el ejercicio del liderazgo para una gestión efectiva de la calidad de la educación a distancia, es una tarea compleja pero no imposible. Comprende, ante todo, ubicarse en la tridimensionalidad en la cual se desenvuelve la educación a distancia, compuestas por las dimensiones de espacio, tiempo y virtualidad. Igualmente, es necesario que los líderes de la educación a distancia tengan un conocimiento al menos funcional satisfactorio de la tecnología instruccional digital que sirve de base a la actual edición de la educación a distancia y lo seguirá siendo en el futuro, al igual que la manifestación de la calidad en los diversos componentes y dominios de esa tecnología instruccional. Este proceso deberá realizarse en un ambiente en el cual la educación a distancia es aún una innovación que no ha terminado de difundirse en la sociedad. Por ello, la gestión de su calidad implica el ejercicio de un liderazgo que pueda manejar efectivamente los diversos elementos del proceso de difusión de la educación a distancia como innovación y su adopción por diferentes tipos de actores, pertenecientes a diversas categorías sociales con diferentes necesidades, aspiraciones y expectativas. 


\section{REFERENCIAS BIBLIOGRÁFICAS}

Beltrán, J. (1993). Procesos, estrategias y técnicas de aprendizaje. Madrid: Síntesis.

CREAD-UTPL (2004) Memorias del Proyecto sobre Estándares de Calidad de la Educación a Distancia en América Latina y el Caribe. Universidad Técnica Particular de Loja (UTPL) y CREAD. Loja, Ecuador.

Gros Salvat, B. (2000) El ordenador invisible: hacia la apropiación del ordenador en la enseñanza. Barcelona: Gedisa.

Lepeley, M. T. (2001) Gestión y calidad en la educación. New York: McGraw Hill.

Lévy, P. (1997). Cyberculture. Paris: Odile Jacob.

Rogers, E y Agarwala-Rogers, R (1980) La comunicación en las organizaciones. New York: McGraw Hill.

Rogers, E. (2003) Diffusion of Innovations. 5a. edición. New York: Free Press.

Schlosser, C y Burmeister, M (1999) Best of both worlds: the NOVA Southeastern University ITDE Model of Distance Education. Revista Tech Trends, V. 43, Issue 5, pp 45-48.
Seels, B. y Richey, R. (1994) Instructional Technology: the definitions and domain of the field. Publications of the Association for Educational Communications and Technology (AECT) Washington, D.C., USA.

Silvio, J. (2000) La Virtualización de la Universidad: ¿cómo transformar la educación superior con la tecnología? Ediciones IESALC-UNESCO. Caracas, Venezuela [en línea]. Disponible en: http:// www.iesalc.unesco.org.ve/prog ramas/internac/ univ virtuales/venez uela/ La virtualizacion univ.pdf [consulta 2005, 28 de septiembre].

Silvio, J . (2004) Hacia la articulación del aprendizaje virtual y no-virtual. 3er Congreso Internacional sobre Docencia Universitaria e Innovación. 30 de Junio al 2 de Julio, 2004. Gerona, España

Silvio, J. (2005) Hacia una educación virtual de calidad, pero con equidad y pertinencia. Virtual Educa 2005. México, 20-24 de J unio, 2005.

Valente, T. (1995) Network Models of the Diffusion of Innovations. Cresskill, NJ :Hampton Press.

Weiser, M. (1996) Ubiquitous Computing [en línea]. Disponible en: http:// www.ubiq.com/hypertext/ weis er/UbiHome.html [consulta 2005, 28 de septiembre]. 


\section{PALABRAS CLAVE}

Liderazgo en la Educación a Distancia, Gestión de la Calidad de la Educación a Distancia, Difusión de la Educación a Distancia como Innovación.

\section{KEY WORDS}

Leadership in Distance Education, Management of Quality of Distance Education, Diffusion of Distance Education as an Innovation.

\section{PERFIL ACADÉMICO DEL AUTOR}

J osé Silvio, de nacionalidad venezolana, profesor de la Nova Southeastern University de Estados Unidos de América en el Programa de Doctorado en Tecnología Instruccional y Educación a Distancia. Es jubilado de la UNESCO y en ella desempeñó puestos internacionales en Francia y Venezuela. Igualmente, ha sido Profesor de la Universidad Central de Venezuela. Es Licenciado en Sociología de la Universidad Central de Venezuela y Doctor en Ciencias de la Educación de la Universidad de Paris. Ha realizado cursos de perfeccionamiento en Informática y Telemática aplicadas a la educación a distancia en universidades de Francia y Canadá. Su tema principal de interés profesional comprende la utilización de la informática y la telemática en la educación. Es autor individual y editor de varios libros y artículos sobre ese tema.

Dirección postal:

4440 NW 73rd Av. \#CCS-1229

Miami, FL 33166

USA

E-mail: jsilvio@nova.edu

Fecha recepción del artículo: 21. 03. 2006

Fecha aceptación del artículo: 07. 04. 2006 